\title{
Thermally Activated Processes at the Co/ZnO Interface Elucidated Using High Energy X-rays
}

\author{
Jacques A. Dumont, ${ }^{* \dagger}$ Mac C. Mugumaoderha, ${ }_{+}^{\dagger}$ Jacques Ghijsen, ${ }^{\dagger}$ Sebastian Thiess, ${ }^{\neq}$Wolfgang Drube, ${ }^{\neq}$ \\ Bente Walz, ${ }^{\ddagger}$ Martin Tolkiehn, ${ }^{\ddagger}$ Dmitri Novikov, ${ }^{\ddagger}$ Frank M. F. de Groot, ${ }^{\uparrow}$ and Robert Sporken ${ }^{\dagger}$ \\ ${ }^{\dagger}$ Centre PMR, Facultés Universitaires Notre-Dame de la Paix, rue de Bruxelles 61, B-5000 Namur, Belgium \\ ${ }^{\ddagger}$ Deutsches Elektronen-Synchrotron (DESY), Notkestrasse 85, D-22603 Hamburg, Germany \\ 'Department of Inorganic Chemistry and Catalysis, Utrecht University, Sorbonnelaan 16, 3584 CA Utrecht, The Netherlands
}

\begin{abstract}
A detailed picture of the thermally activated processes occurring at the $\mathrm{Co} / \mathrm{ZnO}$ interface is obtained by a combination of high energy X-ray based techniques: X-ray photoelectron and absorption spectroscopies and the kinematical X-ray standing wave method. At room temperature, the growth of a few monolayers of cobalt proceeds by the nucleation of nanometer-sized clusters on the polar oxygen-terminated $(000 \overline{1})$ surface of a $\mathrm{ZnO}$ single crystal. Progressive annealing from 600 to $970 \mathrm{~K}$ allows separating the various interfacial reactions. At the lowest annealing temperature, Co clusters

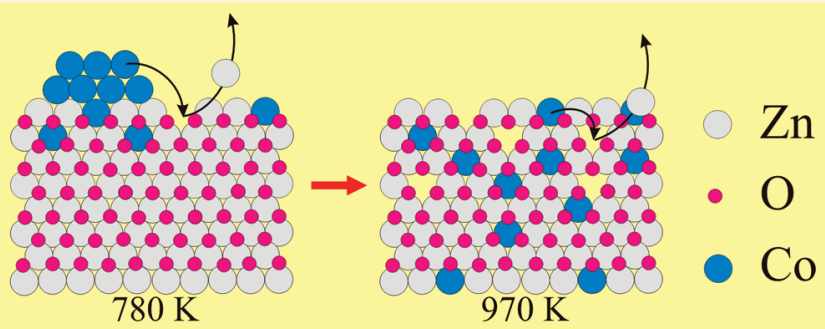
coalesce while keeping their metallic character. Above $700 \mathrm{~K} \mathrm{Co}^{0}$ is gradually oxidized to $\mathrm{Co}^{2+}$ and a thin Co rich ( $\left.\mathrm{Zn}, \mathrm{Co}\right) \mathrm{O}$ layer is formed. It is observed that rock salt $\mathrm{CoO}$ phases may form at the surface when the initial Co thickness exceeds $1 \mathrm{~nm}$. At the highest annealing temperature $(970 \mathrm{~K})$, Co diffuses deeper into $\mathrm{ZnO}$ and $\mathrm{Zn}$ vacancies are created at subsurface sites that were previously occupied by Co.
\end{abstract}

\section{INTRODUCTION}

Most thermodynamic and transport phenomena that are important for technological applications depend on the electronic and chemical properties of buried interfaces. Conventional $\mathrm{X}$-ray photoelectron spectroscopy (XPS) is a key method to study the complex chemistry of solid surfaces. However, its ability to probe electronic properties below the surface is very limited due to the rather short electron escape depth. This limitation can be overcome by using considerably higher-energy photons in XPS, which offers the ability to gain a clearer understanding of the chemical and electronic properties of interfaces several nanometers below the surface. Additionally, it is often desirable to know the crystal symmetry and binding coordination of atoms of interest. This can be achieved by combining highenergy XPS with X-ray absorption near-edge spectroscopy (XANES) and kinematical X-ray standing wave (KXSW) techniques.

$\mathrm{ZnO}$ based materials constitute the building blocks of many actual and potential future technologies. Metal (especially $\mathrm{Cu}$ ) particles grown on $\mathrm{ZnO}$ are excellent catalysts. ${ }^{1}$ (Zn,TM)O ternary compounds where TM is a transition metal, are room temperature diluted magnetic semiconductors (DMS) and can possibly be used for spin current injection in spintronic devices. ${ }^{2}$ Promising technological breakthrough in the field of ultrahigh density data storage as well as high speed electronics may emerge from the two-dimensional electron gas (2-DEG) at the interface between oxides such as $\mathrm{ZnO}$ and $(\mathrm{Zn}, \mathrm{Mg}) \mathrm{O} .^{3}$
The goal of this work is 2-fold: at first, we aim at contributing to a deeper understanding of the structural and electronic properties of the $\mathrm{Co} / \mathrm{ZnO}$ system. Very recently, thermal oxidation and diffusion of $\mathrm{Co}$ into polar $\mathrm{ZnO}$ surfaces was demonstrated; ${ }^{4,5}$ nevertheless, it is still unclear whether $\mathrm{Co}^{2+}$ is incorporated into a $\mathrm{CoO}$ secondary phase or is located in substitutional sites in the $\mathrm{ZnO}$ wurtzite lattice. In the present work, XPS is used both to probe the chemical reactions between $\mathrm{Co}$ and $\mathrm{ZnO}$ and, in particular, the oxidation of $\mathrm{Co}$ and to monitor the spatial distribution of $\mathrm{Co}, \mathrm{Zn}$, and $\mathrm{O}$ in the surface region as well as the stoichiometry of $\mathrm{ZnO}$. Most importantly, the simulation of the $\mathrm{Co}^{2+}$ XPS spectra using charge-transfer multiplet calculations together with results from the XANES and KXSW analysis allows us to determine whether Co oxide precipitates into $\mathrm{CoO}$ rock salt phases or dilutes in the $\mathrm{ZnO}$ lattice. The second aim consists of proposing a way to tune the structural and electronic properties of the $\mathrm{Co} / \mathrm{ZnO}$ system, which could then be applied to other metal/oxide interfaces. Thanks to processes activated by annealing, nanometer scale clusters grown on $\mathrm{ZnO}$ merge into larger islands. Further annealing induces the diffusion of $\mathrm{Co}$ into $\mathrm{ZnO}$ where it substitutes $\mathrm{Zn}$ atoms. The concentration profile of Co into the $\mathrm{ZnO}$ lattice is also tunable by the annealing temperature.

\footnotetext{
Received: September 14, 2010

Revised: February 18, 2011

Published: March 24, 2011
} 


\section{EXPERIMENT}

Experimental Methods. The $(000 \overline{1})$ surface of a $\mathrm{ZnO}$ single crystal produced by pressurized melt growth was polished by a mechanical and chemical method (NovaSic). The sample was then ultrasonically cleaned in dimethylsulfoxide and acetone and blown dry with nitrogen after each bath. After being loaded into an ultrahigh vacuum (UHV, base pressure $\approx 2 \times 10^{-9} \mathrm{mbar}$ ), it was cleaned by cycles of $\mathrm{Ar}^{+}$sputtering $(1 \mathrm{keV}, 5 \mathrm{~min})$ and annealing (925 K, $20 \mathrm{~min}$ ) until no more contaminants were detected by XPS and a sharp LEED pattern was observed. The sample was heated by direct current flow through a glassy carbon wafer in contact with the single crystal. The temperature was calibrated using a $\mathrm{K}$ type thermocouple in contact with the $\mathrm{ZnO}$ surface prior to cobalt deposition. Co (99.999\% purity) was deposited from an electron beam evaporator. The deposition rate measured using a quartz oscillator before each deposition was between 0.016 and $0.075 \mathrm{~nm}$ per minute. The Co coverage is specified as equivalent thickness, i.e., as the thickness of an equivalent uniform layer of bulk Co with the same mass. The experimental data collected on the $\mathrm{Co} / \mathrm{ZnO}$ system were also compared to measurements performed on a $\mathrm{CoO}$ single crystal, which was cleaned by $\mathrm{Ar}^{+}$sputtering $(1 \mathrm{keV}, 40 \mathrm{~min})$.

The chemical interactions between $\mathrm{Zn}, \mathrm{Co}$, and $\mathrm{O}$ and the spatial distribution of these elements in the sample were studied by XPS and XANES. These measurements were performed at the X-ray wiggler beamline BW2 at HASYLAB, Hamburg, Germany. ${ }^{6,7}$ For XPS, a photon energy of $3500 \mathrm{eV}$ with a bandpass of $460 \mathrm{meV}$ was selected. Photoelectrons were collected in normal emission geometry with the analyzer mounted $45^{\circ}$ with respect to the polarization direction of the linearly polarized X-ray beam. The Au $4 f_{7 / 2}$ peak at $84.0 \mathrm{eV}$ binding energy $(\mathrm{BE})$ recorded from a reference sample was used to calibrate the binding energy scale. To account for possible photon energy drifts due to changing heat load on the X-ray optical components, reference spectra were recorded before and after each core level and valence band data set. XANES measurements were performed by recording the total photoelectron yield; i.e., the sample drain current.

The position that $\mathrm{Co}$ atoms occupy in the $\mathrm{ZnO}$ host lattice was studied with the KXSW method. ${ }^{8,9}$ The method is based on the properties of diffraction scattering and makes use of the X-ray standing wave, formed inside a crystal by coherent interference of incoming and Bragg diffracted waves. ${ }^{10}$ In general, the standing wave period is equal to that of the diffracting planes and the phase depends on the deviation from the exact Bragg condition. The incident X-rays excite atoms inside the crystal and their fluorescence yield $Y$ is proportional to the $\mathrm{X}$-ray field intensity at the atom location. The movement of the nodes and antinodes of the wave field that follows the crystal rotation leads to variation of the fluorescence yield, and the form and amplitude of this variation can be used to detect the emitting atoms' positions in the host lattice unit cell. Only atomic shells with absorption edges below the energy of the incident beam $E_{0}$ contribute to the fluorescence signal, however, for a KXSW experiment $E_{0}$ can be freely chosen.

The $\mathrm{ZnO}$ crystals were not perfect enough for the conventional XSW approach, as the fast signal variation in the vicinity of the Bragg angle is blurred due to the mosaicity of the sample. Therefore, we have used the new kinematical XSW method, which uses the weak wave fields formed at large deviation from the Bragg condition. The method is very tolerant to lattice imperfections.

$\mathrm{X}$-ray detector

Fluorescence detector

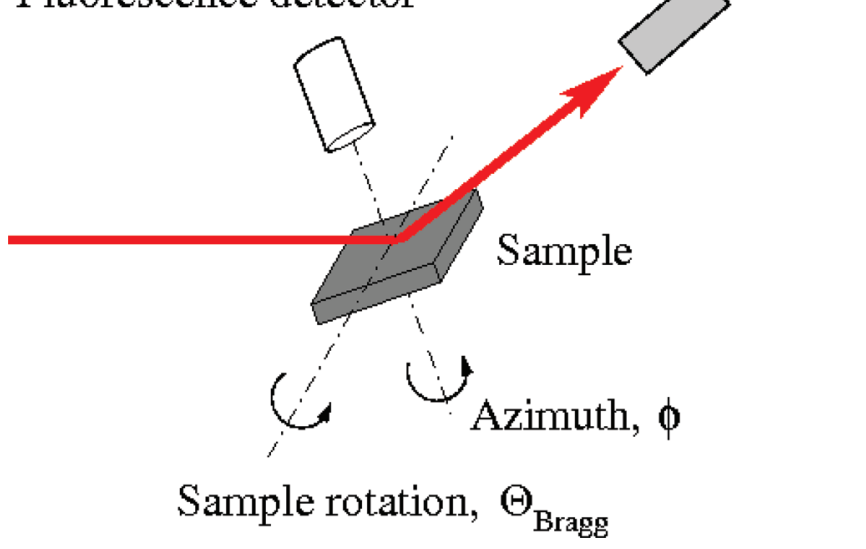

Figure 1. Schematic layout of the KXSW experiment at BW1. The normal to the Bragg scattering planes is aligned along the azimuthal rotation axis.

The KXSW measurements were carried out at the undulator beamlines BW1 at HASYLAB, Hamburg, Germany and ID32 at ESRF, Grenoble, France. A double-crystal Si(111) monochromator was used for wavelength tuning to the X-ray energies $E_{0}=$ $9.7 \mathrm{keV}$ above the $\mathrm{Zn} \mathrm{K}$ and $\mathrm{Co} \mathrm{K}$ absorption edges and $E_{0}=9.1$ $\mathrm{keV}$ below the $\mathrm{Zn}$ K-edge for refinement of the cobalt atom position. The higher harmonics were suppressed by mirrors. A multiple-circle diffractometer allowed sample alignment with rotation around the Bragg plane reciprocal lattice vector for control of multiple reflection effects. ${ }^{8}$ The Bragg scattering was detected with a scintillation counter, secondary fluorescence with a VORTEX silicon drift detector (Figure 1).

Theoretical Methods. Co $2 \mathrm{p}$ XPS spectra from cobalt monoxide in tetrahedral (wurtzite) and octahedral (rock salt) symmetry were simulated using the charge-transfer multiplet (CTM) approach which is described in detail elsewhere. ${ }^{11}$ Calculations were performed using the CTM4XAS user interface. ${ }^{12}$ This program is based on the Anderson model of the charge-transfer multiplets and provides simulation of X-ray absorption, photoelectron spectra of transition metal compounds. The calculated spectra were convoluted with a $0.3 \mathrm{eV}$ wide (fwhm) Lorentzian to account for the core hole lifetime as well as with a $0.3 \mathrm{eV}$ wide (fwhm) Gaussian to simulate the experimental energy resolution.

\section{RESULTS}

General Observations. Zn 2p, O 1s, and Co $2 \mathrm{p}_{3 / 2}$ XPS core level lines have been recorded at successive stages of the experiment (Figure 2). The relative energy alignment of the Zn 2 p peaks compensates for band bending effects occurring upon cobalt deposition and allows for a clearer identification of changes in the peak line shape.

The line shape of the $\mathrm{Zn} 2 \mathrm{p}$ peak is not affected by the Co deposition and subsequent annealing. At higher binding energies weak satellites are observed (see inset in Figure 2) thanks to the high bulk sensitivity of high energy XPS. These features are attributed to bulk-related electronic excitation: ${ }^{13,14}$ features $\mathrm{A}, \mathrm{B}$, and $\mathrm{C}$ are attributed to interband transitions from the filled $\mathrm{O} 2 \mathrm{p}$ and $\mathrm{Zn} 3 \mathrm{~d}$ bands to the empty density of states in the conduction band while feature $\mathrm{D}$ is assigned to a collective excitation 

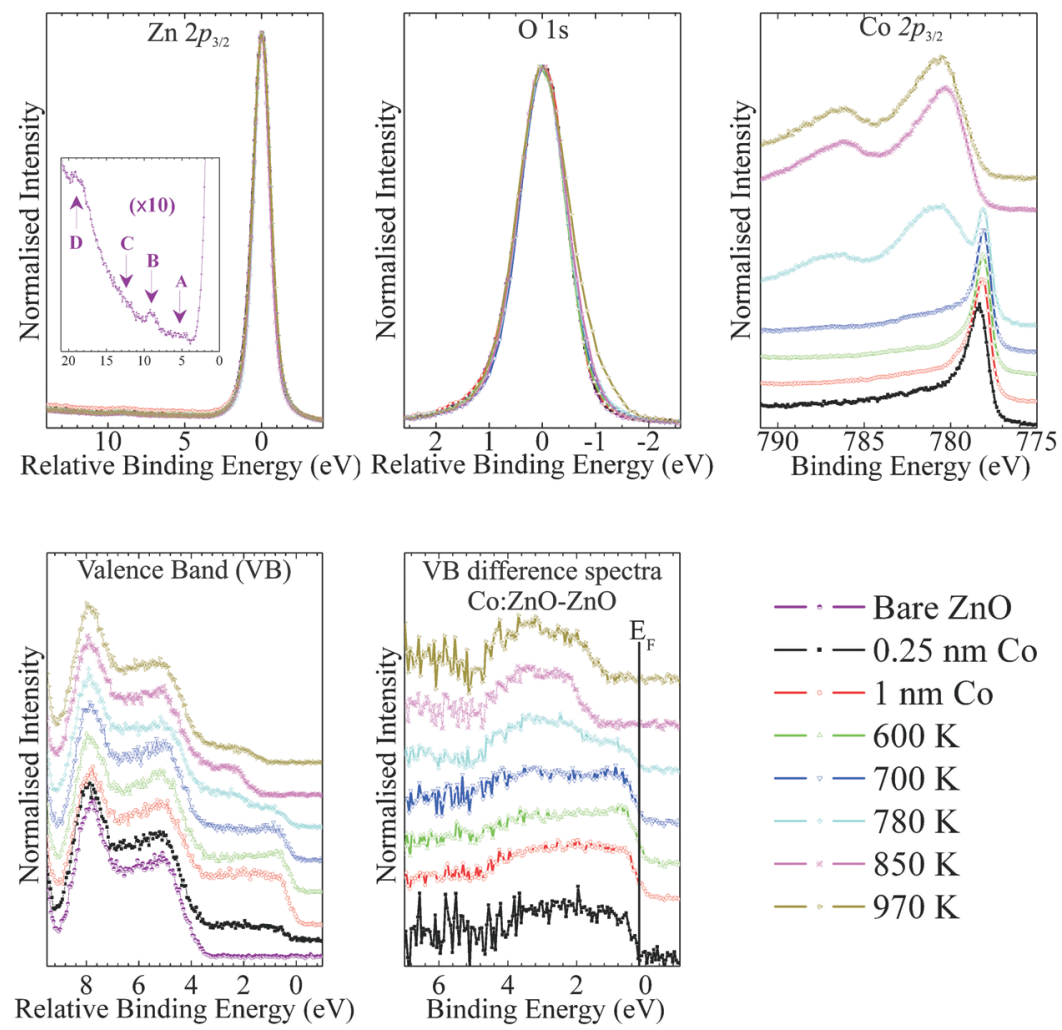

$$
\begin{aligned}
& -\cdot-\text { Bare } \mathrm{ZnO} \\
& -\cdot-0.25 \mathrm{~nm} \mathrm{Co} \\
& -\cdot-1 \mathrm{~nm} \mathrm{Co} \\
& --600 \mathrm{~K} \\
& --700 \mathrm{~K} \\
& --780 \mathrm{~K} \\
& --850 \mathrm{~K} \\
& --970 \mathrm{~K}
\end{aligned}
$$

Figure 2. XPS spectra from $\mathrm{Zn} 2 \mathrm{p}, \mathrm{O} 1 \mathrm{~s}$, Co $2 \mathrm{p}$ core levels and the $\mathrm{ZnO}$ valence band at successive stages of the experiment. The $\mathrm{Zn} 2 \mathrm{p}$ peak positions were aligned relative to their intensity maximum and an identical shift was applied to the corresponding $\mathrm{O}$ 1s peak. The inset shows the Zn $2 \mathrm{p}$ spectral region on an expanded scale. Features A, B, C, and D are described in the text. In valence band spectra, the $\mathrm{Zn} 3 \mathrm{~d}$ related features (located $10.7 \mathrm{eV}$ below $E_{\mathrm{F}}$ ) were aligned on their maximum intensity to compensate for band bending effects. The last panel shows difference spectra obtained by subtracting a weighted valence band spectrum of bare $\mathrm{ZnO}$ from the aligned successive spectra (see text for details).

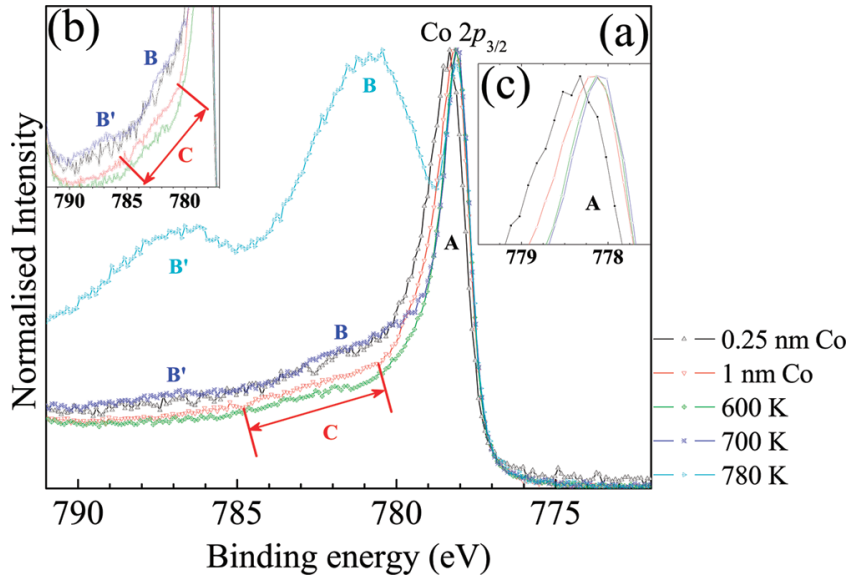

Figure 3. Co $2 \mathrm{p}_{3 / 2}$ XPS spectra after initial deposition and annealing. Insets show $\mathrm{Co} 2 \mathrm{p}_{3 / 2}$ spectral regions on an expanded scale. Features $\mathrm{A}-\mathrm{D}$ are discussed in the text.

(plasmon) of $\mathrm{O} 2 \mathrm{p}$ and $\mathrm{Zn} 4 \mathrm{~s}$ electrons. The line shape of $\mathrm{O} 1 \mathrm{~s}$ spectra is also largely unaffected by the presence of cobalt except for a low energy component which appears upon annealing at $970 \mathrm{~K}$.

During the growth process as well as after annealing at $600 \mathrm{~K}$, the Co $2 \mathrm{p}$ line shape corresponds to that of metallic cobalt. Oxidation is initiated upon annealing at $700 \mathrm{~K}$ and results in several modifications of the Co $2 \mathrm{p}$ spectrum: a $3 \mathrm{eV}$ shift to higher $\mathrm{BE}$, a significant line broadening and the appearance of a pronounced charge-transfer satellite (at a BE of $787 \mathrm{eV}$ ), which will be discussed in more detail below. Metallic and oxidized cobalt coexist after annealing at 700 and $780 \mathrm{~K}$ but only oxidized Co remains at higher temperature.

The evolution of the valence band at the different stages of the experiment is also represented in Figure 2. As observed for $\mathrm{Zn} 2 \mathrm{p}$, the line shape of $\mathrm{Zn}$ related features in the valence band is not affected by the experiment. To compensate for band bending effects, the spectra were aligned relative to the $\mathrm{Zn} 3 \mathrm{~d}$ (not shown) peaks of bare $\mathrm{ZnO}$ appearing at a $\mathrm{BE}$ of $10.7 \mathrm{eV}$ with respect to the Fermi level. This procedure allows us to better highlight the remaining spectral shape modifications and to extract these changes numerically from the raw data: difference spectra were derived by subtracting the valence band spectrum from the bare $\mathrm{ZnO}$ surface after suitable intensity scaling such that the difference is non-negative over the energy range of interest. This crude operation enhances the visibility of modifications to the valence electronic structure. Finally, the resulting difference spectra are presented in the last panel of Figure 2 using their original energy scale referred to the Fermi level.

As can be seen in valence band spectra, the metallic character of the Co density of states, which extends to the Fermi level, is preserved even after annealing at $700 \mathrm{~K}$. At $780 \mathrm{~K}$ signatures from both metallic $\mathrm{Co}$ and $\mathrm{Co}^{2+}$ are observed. At higher temperatures, the valence band edge (Co $3 \mathrm{~d}$ ) moves away from the Fermi level by $1 \mathrm{eV}$, which is characteristic of $\mathrm{Co}^{2+}$ in $\mathrm{ZnO}^{15}$ Finally, the width of the $\mathrm{Co} 3 \mathrm{~d}$ band in $\mathrm{Co}^{2+}$ seems to increase again after the 

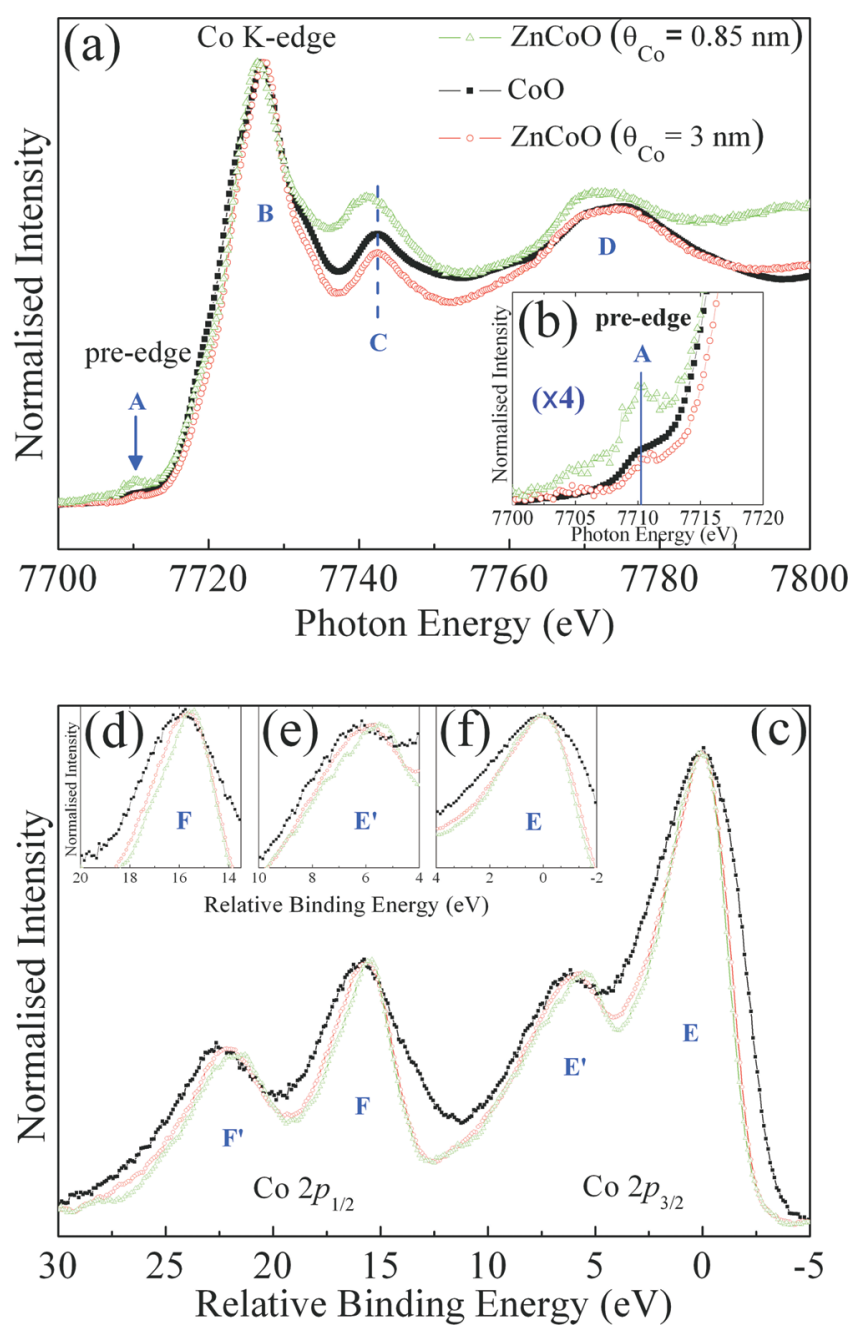

Figure 4. Comparison of Co K-edge XANES and Co 2p XPS spectra from a $\mathrm{CoO}$ single crystal and Co films (thickness 0.85 and $3 \mathrm{~nm}$ ) grown on $\mathrm{ZnO}(000 \overline{1})$ and annealed at $970 \mathrm{~K}$.

final annealing $(970 \mathrm{~K})$ where also a low $\mathrm{BE}$ component in the $\mathrm{O}$ 1s line shape is observed. As will be shown below, these two phenomena may have the same origin but cannot be solely associated to the oxidation of cobalt, which already occurs at much lower temperature (above $700 \mathrm{~K}$ ). Additional experimental results will be provided in the following, which will help to interpret these two observations and to clarify the thermodynamical processes occurring during the annealing.

Structure of the Metallic Co Film. A close inspection of the Co $2 p$ core level data reveals spectral information, helping us to understand how $\mathrm{Co}$ interacts with $\mathrm{ZnO}$. Indeed, as explained in the following, the $\mathrm{BE}$ and width of the Co $2 \mathrm{p}_{3 / 2}$ peak are both tightly related to size effects. At first, from Figure $3 a$ and insets $b$ and $c$ it is obvious that the Co $2 p$ spectrum obtained after deposition of $0.25 \mathrm{~nm}$ is not only significantly broader than other curves (Figure 3 and inset c) but also shifted to higher BE. This is also often observed in photoemission from few-atom clusters and can be related to a weaker screening of the photohole. ${ }^{16}$ After Co deposition of $1 \mathrm{~nm}$, the Co $2 \mathrm{p}_{3 / 2}$ peak narrows and shifts to lower $\mathrm{BE}$. This trend proceeds even further upon annealing at $600 \mathrm{~K}$, indicating a gradual loss of the size effect along these successive steps.
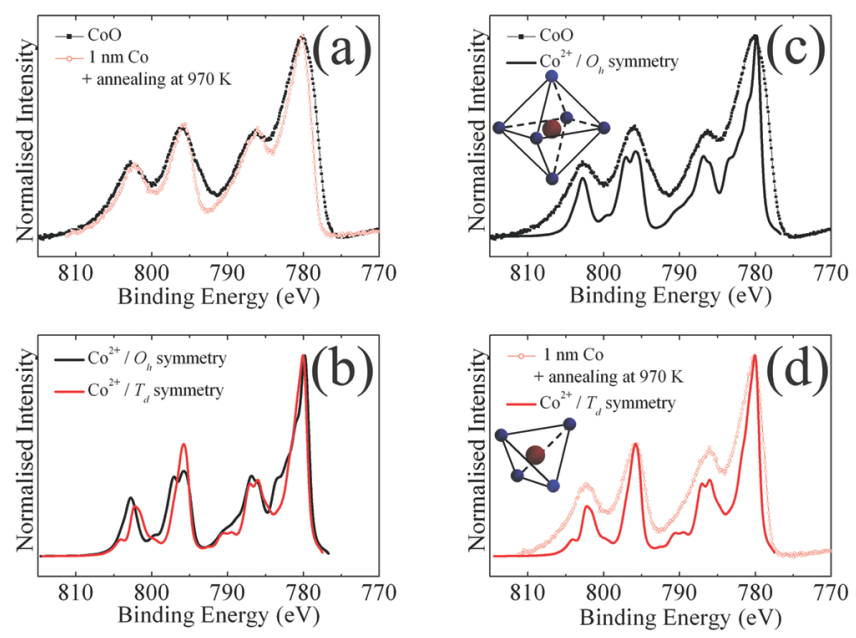

Figure 5. (a) Co $2 \mathrm{p}$ XPS spectra from a CoO single crystal and a $1 \mathrm{~nm}$ thick Co film grown on $\mathrm{ZnO}(000 \overline{1})$ and annealed at $970 \mathrm{~K}$. The experimental spectra are compared to theoretical XPS spectra calculated for octahedral (c) and tetrahedral (d) coordination of the $\mathrm{Co}^{2+}$ ion. The comparison of both calculated spectra is shown in (b).

The spectral region indicated by $\mathrm{C}$ in Figure $3 \mathrm{a}$ and inset $\mathrm{b}$ corresponds to the location where a satellite was reported by Nath et al., ${ }^{17}$ which is related to the existence of different channels for screening the hole from the Co $2 p$ level. This low-intensity feature is characteristic of small clusters and not observed for bulk materials. ${ }^{18}$ In our case, it appears to be very weak in spectra from samples with low Co coverage and to disappear altogether after annealing.

In summary, the first three stages of this experiment indicate that nanometer sized clusters are initially formed, likely due to the poor adhesion of $\mathrm{Co}$ onto $\mathrm{ZnO}$. The successive reduction and disappearance of size effects in the spectra are indicative of larger clusters, i.e., that the clusters coalesce upon the increase in Co amount and annealing temperature. These observations are in good agreement with a previous work from our group where the nucleation of Co clusters on $\mathrm{ZnO}$ and their coalescence upon annealing was observed by STM. ${ }^{19}$

Annealing at $700 \mathrm{~K}$ leads to the appearance of two satellites labeled $\mathrm{B}$ and $\mathrm{B}^{\prime}$ (Figure 3 and inset $\mathrm{b}$ ) attributed to the beginning of the oxidation process, since these satellites appear at the same energy as the main lines from cobalt in the $2+$ oxidation state which becomes dominant after annealing at $780 \mathrm{~K}$. The oxidation of Co is discussed in the following section.

Co Oxidation. In the present study, the Co oxide formed by annealing at a temperature higher than $600 \mathrm{~K}$ contains cobalt in a $2+$ oxidation state as in $\mathrm{CoO}$. This is concluded from the similar line shapes of the Co K-edge XANES and Co 2p XPS spectra (Figure 4a,c) from Co films grown on $\mathrm{ZnO}$ and annealed at $970 \mathrm{~K}$ (named $(\mathrm{Zn}, \mathrm{Co}) \mathrm{O}$ in the following) and from a $\mathrm{CoO}$ single crystal.

In Figure 4a, the Co K-edge XANES spectra from two (Zn, Co)O films (prepared with an initial Co amount of $0.85 \mathrm{~nm}$ respectively $3 \mathrm{~nm}$ ) are compared to the spectrum from a CoO single crystal. The pre-edge feature A (inset b) arises from 1 s to $3 \mathrm{~d}$ multipole transitions mediated by the Co $3 \mathrm{~d}-\mathrm{O} 2 \mathrm{p}$ covalency. The absence of any additional feature in the pre-edge indicates that no metallic Co is present in the samples within the detection limit of a few atomic percent. ${ }^{15}$ 
Table 1. Best Parameter Sets Extracted from Charge-Transfer Multiplet Calculations

\begin{tabular}{crrrrrl} 
symmetry & $10 D q$ & $\Delta$ & $U_{\mathrm{dd}}$ & $U_{\mathrm{pd}}$ & $T_{\sigma}$ & $T_{\pi}$ \\
$O_{h}$ & 1.8 & 4.0 & 7.0 & 8.5 & 1.75 & 1 \\
$T_{d}$ & -1.2 & 4.0 & 7.0 & 8.5 & 1 & 1.6 \\
$T_{d}{ }^{21}$ & -0.7 & 5.0 & 6.0 & 8.5 & 1 & 2.16 \\
\hline
\end{tabular}

The Co K-edge XANES spectra of $\mathrm{Co}^{2+}$ in $\mathrm{CoO}$ and $(\mathrm{Zn}$, Co)O are similar, in agreement with Kaspar et al. ${ }^{15}$ Nonetheless, when the spectra obtained for $(\mathrm{Zn}, \mathrm{Co}) \mathrm{O}$ are compared, it appears that in the case of the smaller initial Co amount feature $\mathrm{A}$ is stronger, feature $\mathrm{C}$ is broader and shifted toward lower photon energy while feature $\mathrm{D}$ is narrower. As shown recently, ${ }^{20}$ pre-edge feature $\mathrm{A}$ is much more intense in tetrahedral than in octahedral coordination. Cobalt ions in $\mathrm{CoO}(\mathrm{NaCl}$ structure) are octahedrally coordinated while Co substituting $\mathrm{Zn}$ atoms in $\mathrm{ZnO}$ (wurtzite structure) are tetrahedrally coordinated. This would imply that, when the initial (i.e., prior to annealing) amount of cobalt is relatively small $(\theta \leq 1 \mathrm{~nm})$, Co is thermally incorporated into the $\mathrm{ZnO}$ wurtzite lattice where it substitutes Zn, while for a larger initial Co amount, a secondary rock salt $\mathrm{CoO}$ phase is preferentially formed after annealing.

As seen in Figure $4 c$ (and insets $d-f$ ), the shape of the Co $2 p$ XPS spectra is also affected by the initial Co amount: the apparent spin-orbit splitting (energy separation between feature $\mathrm{E}$ and $\mathrm{F}$ ), the charge-transfer splitting (energy separation between feature $E$ and $E^{\prime}$ ), and the width of the lines all increase for larger initial Co amount. Before providing an interpretation for this trend, the origin of these features is briefly recalled. The oxidation of Co leads to the appearance of a double peak structure due to the existence of multiple final states associated with charge transfer between $\mathrm{Co}$ and neighboring $\mathrm{O}$ ligands. Indeed, the initial electronic configuration of $\mathrm{Co}^{2+}$ is $\mathrm{d}^{7}$. Following the photoemission process, the photohole on the Co $2 p$ core level is screened by valence electrons. The screening may be increased by additional charge transfer from ligand atoms (oxygen) to the cobalt. As a result, multiple final states may coexist, giving rise to satellite structures. The two broad features $E$ and $E^{\prime}$ in Figure $4 c$ reflect the energy range of the principal configurations $\mathrm{cd}^{7}, \mathrm{~cd}^{8} \mathrm{~L}$, and $\mathrm{cd}^{9} \mathrm{~L}^{2}$, where $c$ denotes the core hole, $\mathrm{d}^{n}$ is the presence of $n$ electrons in the $\mathrm{d}$ shell, and $\mathrm{L}^{m}$ is the number $m$ of holes on the ligand 2p orbital after electron transfer. The line shape of features $E$ and $E^{\prime}$, their relative energy splitting, and their intensity ratio is mostly affected by the Co oxidation state. In addition, the crystal field symmetry might also affect the fine details of the line shape since features $E$ and $E^{\prime}$ constitute the envelope of a multiplet structure arising from the various electronic configurations within the open $d$ shell for each of the principal configuration. The Co $3 \mathrm{~d}$ shell is split into low energy $t_{2 g}$ (respectively e) and high energy e $e_{g}$ (respectively $t_{2}$ ) levels for octahedral (respectively tetrahedral) crystal field symmetry. The various possibilities of filling the available d electrons into these levels lead to multiple energy states that can additionally be split by spin-orbit coupling with the core $2 p$ hole.

In the following we aim to establish whether the modifications observed in the Co 2p XPS spectra with increasing Co amount could result from different (i.e., tetrahedral or octahedral) crystal field symmetries around Co atoms. We performed calculations based on the charge-transfer multiplet (CTM) model and compared the experimental spectra (Figure 5a) from a rock salt

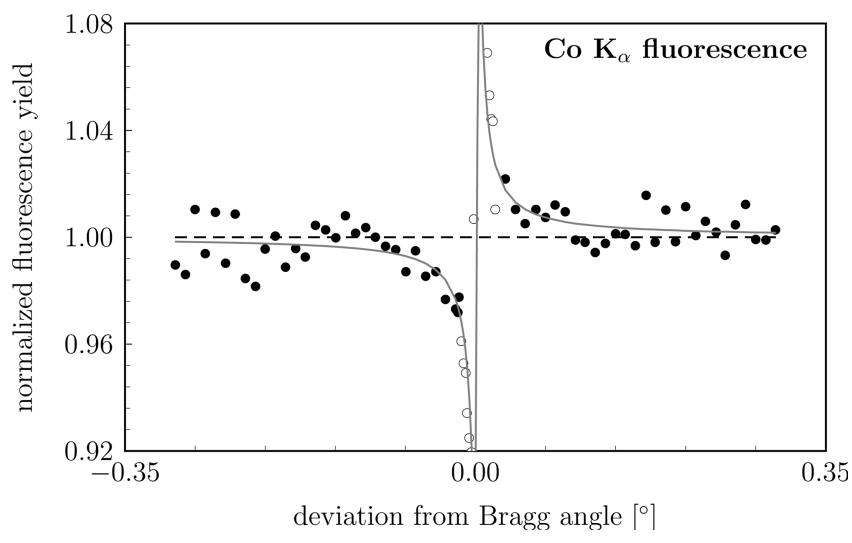

Figure 6. Co K $\alpha$ fluorescence yield (dots) and theoretical curve (solid line) in the vicinity of the $\mathrm{ZnO}$ (002) Bragg reflection at $9.1 \mathrm{keV}$. Only the black data points were used for the kinematical data evaluation.

$\mathrm{CoO}$ single crystal and $(\mathrm{Zn}, \mathrm{Co}) \mathrm{O}$ with the calculated spectra for octahedrally and tetrahedrally coordinated $\mathrm{Co}^{2+}$ (Figure $5 \mathrm{~b}$ ). The $\mathrm{Co}^{2+}$ spectra were calculated using the CTM4XAS program described above, which includes $\mathrm{cd}^{7}$ and $\mathrm{cd}^{8} \mathrm{~L}$ configurations. The finite crystal temperature $(300 \mathrm{~K})$ is included in the calculation through a Boltzmann distribution of the occupation of the initial states. Table 1 contains parameter sets referring to the spectra shown in Figure $5 \mathrm{~b}$ and from Kobayashi's atomic multiplet simulations of Co $2 \mathrm{p}$ spectra obtained on $(\mathrm{Zn}, \mathrm{Co}) \mathrm{O}$ epilayers. ${ }^{21} 10 \mathrm{Dq}$ refers to the crystal field splitting. It takes negative (respectively positive) values for tetrahedral (respectively octahedral) symmetry. $\Delta$ is the chargetransfer parameter, $U_{\mathrm{dd}}$ is the Hubbard $\mathrm{U} \mathrm{d}-\mathrm{d}$ repulsion energy, $U_{\mathrm{pd}}$ is the core hole potential, and $T_{\sigma}$ (respectively $T_{\pi}$ ) is the hybridization energy between the metal $\mathrm{d}_{3 z^{2}-r^{2}}$ or $\mathrm{d}_{x^{2}-y^{2}}$ (respectively $\mathrm{d}_{x y}, \mathrm{~d}_{y z}$ or $\mathrm{d}_{z x}$ ) and the ligand $\mathrm{p}$ orbitals. As shown by Bocquet and Fujimori, $^{22}$ there is no unique set of parameters leading to a proper fit of the experimental data. Hence, to simulate the data in a consistent way, we first tried to match both the intensity ratio and energy splitting between the main $2 \mathrm{p}_{3 / 2}$ line and its charge-transfer satellite. In the case of $\mathrm{O}_{h}$ symmetry, we assume that $\mathrm{Co}^{2+}$ is in a high spin state and refer to Bocquet's look-up tables ${ }^{22}$ to define a set of initial parameters. These parameters are then slightly modified for a better match of our data. The final adjustment consists in tuning the crystal field energy $10 \mathrm{Dq}$ inside a window ranging from 0.5 to $2.0 \mathrm{eV}$. In the case of $T_{d}$ symmetry, we use the best parameter set obtained for $O_{h}$ symmetry as initial parameter set, except that the sign of $10 \mathrm{Dq}$ is changed and that the values for $T_{\pi}$ and $T_{\sigma}$ are exchanged. Finally, we tune the value of $10 \mathrm{Dq}$ and $T_{\pi}$ to reach the best match between the calculated and experimental spectra. The parameter sets extracted from this procedure, although slightly different from Kobayashi's, ${ }^{21}$ are in fair agreement with Bocquet's look-up tables. We notice that the hybridization energy and crystal field splitting are slightly smaller in the $T_{d}$ symmetry.

In the experimental spectra displayed in Figure 5a, there are differences between the $\mathrm{Co} 2 \mathrm{p}$ lines from rock salt $\mathrm{CoO}$ and tetrahedrally coordinated $\mathrm{Co}^{2+}$ ions. As seen in Figure $5 b-d$, the simulated spectra reproduce the experimental trend, especially a slight broadening of the Co $2 \mathrm{p}$ main lines in the $\mathrm{O}_{h}$ symmetry. However, on the basis of this alone, we can hardly conclude for one symmetry or the other.

In summary, the analysis of the experimental and simulated XPS spectra reveals that very little difference exists between the spectral signature from rock salt and wurtzite $\mathrm{Co}^{2+}$. XANES data 


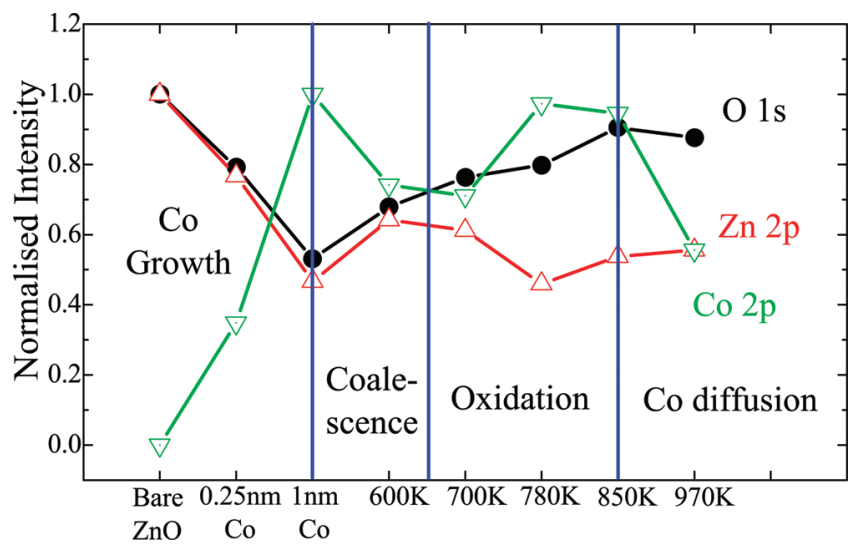

Figure 7. Changes in the $\mathrm{Zn} 2 \mathrm{p}, \mathrm{O} 1 \mathrm{~s}$, and Co $2 \mathrm{p}$ line intensity at the different stages of the experiment. $\mathrm{Zn} 2 \mathrm{p}$ and $\mathrm{O} 1 \mathrm{~s}$ signals are normalized with respect to their value on the bare $\mathrm{ZnO}$ surface. Co $2 \mathrm{p}$ is normalized for $1 \mathrm{~nm}$ Co.

provide a fair indication that for a relatively small initial Co amount, Co atoms are preferentially substituting $\mathrm{Zn}$ atoms in the wurtzite structure. Nonetheless, to further establish the location of the Co atoms, we resorted to KXSW analysis.

Cobalt Atom Position in the $\mathrm{ZnO}$ Lattice. A typical Co $\mathrm{K} \alpha$ fluorescence yield curve is presented in Figure 6.

The experimental data are obtained from a sample after the heat treatment procedure as described above, to ensure that the Co is diffused into the $\mathrm{ZnO}$ and oxidized. The solid line is the fit to the fluorescence yield at the tails of the diffraction curve (black dots). The data in the area where the kinematical diffraction theory cannot be applied (open circles) are excluded from the analysis.

The position of the Co atoms can be found from the fluorescence yield $Y(\Delta \theta)$ :

$$
Y(\Delta \theta) \propto 1+\frac{\Gamma}{\sin 2 \theta_{\mathrm{B}}} Y_{\mathrm{c}} \frac{1}{\Delta \theta}
$$

with the atom position dependent parameter

$$
\mathscr{Y}_{\mathrm{c}}=\left|F_{\mathbf{H}}\right| f_{\mathrm{c}} \cos \left(2 \pi \Phi_{\mathrm{c}}-\arg F_{\mathbf{H}}\right)
$$

where the coherent position $\Phi_{c}$ describes the average position of the impurity atoms measured relative to the diffraction planes and the coherent fraction $f_{\mathrm{c}}$ corresponds to the spread of these atoms around the average position. $\left|F_{\mathbf{H}}\right|$ and $\arg F_{\mathbf{H}}$ are absolute value and phase of the structure factor of $\mathrm{ZnO}$.

The parameter $\mathscr{Y}_{c}=38 \pm 2$ obtained from the experimental data corresponds to $\mathrm{Co}$ atoms occupying the host $\mathrm{Zn}$ atoms position, which is consistent with results obtained by other authors. ${ }^{15}$ The presence of Co atoms at interstitial positions or oxygen sites may thus be excluded, although the experimentally obtained value is lower than the theoretical value $\mathscr{Y}_{c \text {,theo }}=39.95$. Furthermore, it is interesting to calculate the coherent fraction within the single position model (i.e., Co at the $\mathrm{Zn}$ position), which yields $f_{c}=\mathscr{Y}_{c} / \mathscr{Y}_{c \text {,theo }}=0.95 \pm 0.05$. This value is slightly less than unity, which is typical for crystalline matter with a high degree of lattice perfection but small random displacements due to local atomic disorder. The coherent fraction is then identical with the Debye-Waller factor $D_{\mathbf{H}}=\mathrm{e}^{-1 / 2\left\langle(\mathbf{H} \cdot \mathbf{u})^{2}\right\rangle}$. From that a mean displacement $\left(\left\langle u_{z}{ }^{2}\right\rangle\right)^{1 / 2}=0.013 \pm 0.007 \mathrm{~nm}$ of the Co atoms from the $\mathrm{Zn}$ position can be determined. Thus, the KXSW a) bare $\mathrm{ZnO}$

b) $0.25 \mathrm{~nm} \mathrm{Co}$

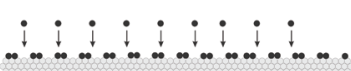

c) $1 \mathrm{~nm} \mathrm{Co}$

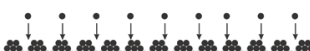

d) $600 \mathrm{~K}$

e) $700 \mathrm{~K}$

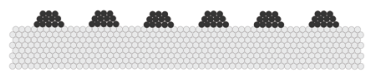

f) $780 \mathrm{~K}$

g) $850 \mathrm{~K}$

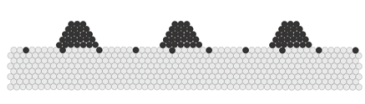

Zn or O

- $\mathrm{Co}$

$-\mathrm{V}_{\mathrm{Zn}}$

h) $970 \mathrm{~K}$

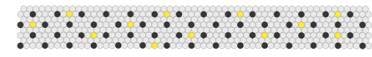

Figure 8. Model showing the various stages of the structural modifications during the growth and annealing of the $\mathrm{Co} / \mathrm{ZnO}$ system.

results confirm the XANES data and additionally justify the choice of atomic configuration models used for the calculated XPS spectra.

Scenario of the $\mathrm{Co} / \mathrm{ZnO}$ Interface Transformation. In the previous sections, several thermally activated processes have been discussed on the basis of the analysis of the XPS and XANES spectral features. Relative intensity changes in the $\mathrm{Zn} 2 \mathrm{p}$, $\mathrm{O}$ 1s, and Co $2 \mathrm{p}$ XPS core level lines (Figure 7) can also be related to reorganization of the film constituents. From the interpretation of these changes and conclusions drawn in the previous sections, we propose a scenario of the film transformation that is explained in the following and illustrated in Figure 8.

Four distinct stages can be identified (Figure 7). At first, the growth of the $1 \mathrm{~nm}$ thick film induces a decrease of the $\mathrm{ZnO}$ related lines and an increase of Co $2 p$ with the amount of Co deposited. As discussed above, at this point the Co film shows signs of size effects that fade out with increasing film thickness. This is attributed to Co aggregation into nanometer scale clusters (Figure $8 \mathrm{a}-\mathrm{c}$ ). Then, after annealing at $600 \mathrm{~K}$, the Co $2 \mathrm{p}$ intensity decreases while the intensities of $\mathrm{O} 1 \mathrm{~s}$ and $\mathrm{Zn} 2 \mathrm{p}$ increase (Figure 7). It was also concluded from the Co $2 p$ line shape that Co remains mostly metallic and the size effects disappear (see above). This indicates that the Co clusters coalesce into larger islands between which the $\mathrm{ZnO}$ surface is again uncovered (Figure $8 \mathrm{~d}-\mathrm{e}$ ).

In the temperature range between 700 and $850 \mathrm{~K}$, the $1: 1 \mathrm{Zn}$ : $\mathrm{O}$ stoichiometry is lost and a depletion of $\mathrm{Zn}$ is observed (Figure 7). At this stage, it is also seen that Co is becoming gradually fully oxidized. Hence, we believe that Co progressively diffuses into the $\mathrm{ZnO}$ subsurface where it substitutes $\mathrm{Zn}$. The substituted $\mathrm{Zn}$ probably migrates toward the surface and desorbs (Figure 8f,g).

Finally, when the annealing temperature exceeds $850 \mathrm{~K}$, the Co $2 p$ signal decreases (Figure 7 ). This may be explained by Co diffusing deeper into $\mathrm{ZnO}$. The bulk solubility of $\mathrm{Co}$ into $\mathrm{ZnO}$ is in principle very low (below $1 \%$ at room temperature ${ }^{23}$ ) but the formation energy of $\mathrm{Co}_{\mathrm{Zn}}$ defects (Co substituting $\mathrm{Zn}$ atoms) 
decreases in the vicinity of the surface, which explains the oxidation process mentioned earlier. ${ }^{24}$ The bulk solubility limit of $\mathrm{Co}$ in $\mathrm{ZnO}$ also increases in relation with the annealing temperature. ${ }^{25}$ Hence, dilution of $\mathrm{Co}$ and its diffusion deeper into the bulk of $\mathrm{ZnO}$ is favored by annealing.

As mentioned above, the $\mathrm{O}$ 1s line shape changes after the final annealing at $970 \mathrm{~K}$. It can therefore not solely be related to the oxidation process, which is already complete at $850 \mathrm{~K}$. In addition, such features in the $\mathrm{O}$ 1s XPS line shape related to Co oxidation have not been reported in the literature about $(\mathrm{Zn}$, $\mathrm{Co}) \mathrm{O}$ ternary compounds. The change in the $\mathrm{O}$ 1s line shape could also be related to the precipitation of $\mathrm{Co}$ into a $\mathrm{CoO}$ phase where each $\mathrm{O}$ atom is bound mostly to Co atoms whereas in diluted $(\mathrm{Zn}, \mathrm{Co}) \mathrm{O}, \mathrm{O}$ atoms are bound to one Co atom at maximum. In such a case, the local electronic structure at the $\mathrm{O}$ atoms in $\mathrm{CoO}$ would be more strongly perturbed by the electronegativity difference between Co and $\mathrm{Zn}$. Nevertheless, the formation of a $\mathrm{CoO}$ rock salt secondary phase was ruled out for such a small $(1 \mathrm{~nm})$ initial Co amount by the present XPS, XANES, and KXSW measurements. Finally, the precipitation of Co into a $\mathrm{CoO}$ wurtzite phase is not compatible with the drop of the Co $2 p$ line intensity observed at $970 \mathrm{~K}$ (Figure 7).

Therefore, the change in the $\mathrm{O} 1 \mathrm{~s}$ line shape at $970 \mathrm{~K}$ requires another interpretation. Diffusion of $\mathrm{Co}$ into $\mathrm{ZnO}$ may proceed in various ways. In the present work, evidence is provided that Co diffuses via $\mathrm{Zn}$ substitution because, above $780 \mathrm{~K}$, Co is always found in a $2+$ oxidation state. Hence, when $\mathrm{Co}^{2+}$ leaves a subsurface site to diffuse deeper into the bulk of $\mathrm{ZnO}, \mathrm{Zn}$ vacancies are likely to be created at the previous Co sites (Figure $8 \mathrm{~h}$ ).

$\mathrm{Zn}$ vacancies constitute very mobile defects under $n$-type doping $^{26}$ and create acceptor levels located $0.9 \mathrm{eV}$ above the $\mathrm{ZnO}$ valence band edge. ${ }^{27}$ As the $\mathrm{ZnO}$ single crystals used in this study are $n$-type, these acceptor levels are filled with electrons. The electronic structure of $\mathrm{O}$ atoms in the vicinity of $\mathrm{Zn}$ vacancies is therefore strongly perturbed: each of the four atoms surrounding this vacancy has an extra half electron. We believe that the low binding energy feature observed on the $\mathrm{O} 1 \mathrm{~s}$ line and the spreading of the valence band edge toward the Fermi level (Figure 2) could both be related to a high density of subsurface $\mathrm{Zn}$ vacancies created after the diffusion of Co deeper into the bulk. However, additional experimental evidence from, e.g., photoluminescence data would be required to confirm this hypothesis.

\section{CONCLUSIONS}

The combination of high energy $\mathrm{X}$-ray based techniques allowed us to elucidate the complex processes occurring when annealing an ultrathin Co film grown on a $\mathrm{ZnO}(000 \overline{1})$ single crystal. When the annealing temperature remains below $700 \mathrm{~K}$, the nanometer scale clusters from the Co film remain metallic and coalesce. Above that temperature, oxidation starts to proceed via $\mathrm{Zn}$ substitution. When the annealing temperature reaches $970 \mathrm{~K}, \mathrm{Co}^{2+}$ ions diffuse deeper into the bulk of the $\mathrm{ZnO}$ single crystal. Following this diffusion, $\mathrm{Zn}$ vacancies are formed in the vicinity of the surface at sites previously occupied by Co atoms. From a technological point of view, this study shows that the structural properties of this metal/oxide interface can be thermally tuned to provide a wide variety of interesting systems (Figure 8) ranging from nanometer scale clusters showing size-dependent electronic structure relevant, e.g., for catalytic applications to ternary metal oxides with various levels of Co concentration. From a more fundamental point of view, a deeper insight has been gained on the processes associated with the substitution of $\mathrm{Zn}$ atoms by $\mathrm{Co}$ and with the dilution of Co into the $\mathrm{ZnO}$ lattice.

\section{AUTHOR INFORMATION}

\section{Corresponding Author}

*E-mail: jacques.dumont@fundp.ac.be.

\section{ACKNOWLEDGMENT}

We thank W. Caliebe for providing a $\mathrm{CoO}$ crystal. This work was supported by the Belgian Fund for Scientific Research (FNRS-FSR), by the Belgian Office for Scientific, Technical and Cultural Affairs (PAI 5.01) and by DESY and the European Community's Seventh Framework Programme (FP7/20072013) under grant agreement no. 226716. J.A.D. and J.G. are respectively postdoctoral researcher and research associate from the Belgian National Fund for Scientific Research (NFSR).

\section{REFERENCES}

(1) Campbell, C. Surf. Sci. Rep. 1997, 27, 1.

(2) Pan, F.; Song, C.; Liu, X.; Yang, Y.; Zeng, F. Mater. Sci. Eng. R 2008, 62, 1 .

(3) Tsukazaki, A.; Ohtomo, A.; Kita, T.; Ohno, Y.; Ohno, H.; Kawasaki, M. Science 2007, 315, 1388.

(4) Hyman, M.; Martono, E.; Vohs, J. J. Phys. Chem. C 201010.1021/ jp9103883.

(5) Xie, W.; Xie, F.; Yu, X.; Xue, K.; Xu, J.; Chen, J.; Zhang, R. Appl. Phys. Lett. 2009, 95, 262506.

(6) Drube, W.; Schulte-Schrepping, H.; Schmidt, H.-G.; Treusch, R.; Materlik, G. Rev. Sci. Instrum. 1995, 66, 1668.

(7) Drube, W. Nucl. Instrum. Methods Phys. Res., Sect. A 2005, $547,87$.

(8) Tolkiehn, M.; Novikov, D.; Fanchenko, S. Phys. Rev. B 2005, 71,165404 .

(9) Tolkiehn, M.; Novikov, D. V. The X-ray Standing Wave Technique: Principles and Applications; World Scientific: Singapore, 2010; in production.

(10) Zegenhagen, J. Surf. Sci. Rep. 1993, 18, 202.

(11) de Groot, F.; Kotani, A. Core Level Spectroscopy of Solids; Taylor \& Francis Group, CRC Press: Bocca Raton FL, 2008.

(12) Stavitski, E.; de Groot, F. M. F. Micron 2010, 41, 687.

(13) Hengehold, R.; Almassy, R.; Pedrotti, F. Phys. Rev. B 1970, 1,4784 .

(14) Dorn, R.; Lüth, H.; Büchel, M. Phys. Rev. B 1977, 16, 4675.

(15) Kaspar, T. C.; Droubay, T.; Heald, S. M.; Nachimuthu, P.; Wang, C. M.; Shutthanandan, V.; Johnson, C. A.; Gamelin, D. R.; Chambers, S. A. New J. Phys. 2008, 10, 055010.

(16) Citrin, P.; Wertheim, G. Phys. Rev. B 1983, 27, 3176.

(17) Nath, K.; Haruyama, Y.; Kinoshita, T. Phys. Rev. B 2001, $64,245417$.

(18) Klebanoff, L. E.; Van Campen, D. G.; Pouliot, R. J. Phys. Rev. B 1994, 49, 2047.

(19) Dumont, J.; Mugumaoderha, M. C.; Seldrum, T.; Frising, F.; Moisson, C.; Turover, D.; Sporken, R. J. Vac. Sci. Technol. B 2007, $25,1536$.

(20) de Groot, F.; Vankó, G.; Glatzel, P. J. Phys.: Condens. Matter 2009, 21, 104207.

(21) Kobayashi, M.; Ishida, Y.; Hwang, J.; Mizokawa, T.; Fujimori, A.; Mamiya, K.; Okamoto, J.; Takeda, Y.; Okane, T.; Saitoh, Y.; Muramatsu, Y.; Tanaka, A.; Saeki, H.; Tabata, H.; Kawai, T. Phys. Rev. B 2005, 72, 201201. 
(22) Bocquet, A.; Fujimori, A. J. Electron Spectrosc. Relat. Phenom. 1996, 82, 87.

(23) Bates, C.; White, W.; Roy, R. J. Inorg. Nucl. Chem. 1966, $28,397$.

(24) Chen, X.; Huang, D.; Deng, W.-J.; Zhao, Y.-J. Phys. Lett. A 2009, 373, 391.

(25) Straumal, B.; Mazilkin, A.; Protasova, S.; Myatiev, A.; Straumal, P.; Baretzky, B. Acta Mater. 2008, 56, 6246.

(26) Huang, G.-Y.; Wang, C.-Y.; Wang, J.-T. Solid State Commun. 2009, 149, 199.

(27) Janotti, A.; Van de Walle, C. J. Cryst. Growth 2006, 287, 58. 